\title{
User Cooperative Mobility for Better Multimedia Communication Quality
}

\author{
Tutomu Murase \\ NEC Corporation \\ 1753 Shimonumabe Nakaharaku \\ Kawasaki JAPAN \\ +81-44-431-7637 \\ t-murase@ap.jp.nec.com
}

\begin{abstract}
For mobile multimedia communications, Quality of Service (QoS) is significantly important. In general it will incur costs to improve QoS. Regarding utility function of cost versus gain for an individual user, it is useful to evaluate relationship between cost such as energy consumption and gain such as QoS. Network operators or service providers could invest more resource facilities to improve QoS that will result in rising users' service fees. User cooperative mobility is one of key technology that do not select between cost and gain tradeoff, but to provide the best solution.
\end{abstract}

\section{Categories and Subject Descriptors}

C.2.1 [Computer-Communication Networks]: Network Architecture and Design-Wireless communication.

\section{General Terms}

Algorithms, Management, Measurement, Performance, Design, Economics, Reliability, Experimentation, Human Factors.

\section{Keywords}

User cooperation, Mobility, Multimedia, Quality of Service, Utility function, Quality of Experience, Mobile computing.

\section{INTRODUCTION}

Demand for multimedia communication is rapidly increasing. So far, mobile users requested networks to improve network Quality of Service (QoS) such as throughput, delay and loss rate for multimedia communication quality. Monthly fees of the users could rise up because of increasing CAPEX and OPEX of networks. From networks point of view, it is interesting to reduce CAPEX and OPEX while keeping high QoS. Mobile users should do something to improve their QoS by themselves. User cooperative mobility is a key control for the improvement of QoS without increasing the monthly fees. User cooperative mobility includes user behaviors such as user movement, movement of their portable access points, and selecting alternate roads/trainlines, with the criteria of obtaining higher radio signal, more

Permission to make digital or hard copies of part or all of this work for personal or classroom use is granted without fee provided that copies are not made or distributed for profit or commercial advantage and that copies bear this notice and the full citation on the first page. Copyrights for third-party components of this work must be honored. For all other uses, contact the owner/author(s). Copyright is held by the author/owner(s).

FhMN'13, August 16, 2013, Hong Kong, China.

ACM 978-1-4503-2183-9/13/08. access point accesses, less radio interferences, and more opportunity of link aggregation.

This is so-called a user cooperation to networks. However, unlike traditional cooperation such as wireless relay communication, the cooperation is done not for others' benefits but for the users'.

It is very natural for users to move so as to obtain higher QoS if the QoS is strongly deserved [3]. However, it is not, if the movement cost is more than the gain results from the move. In general it is difficult to decide movement for mobility or not because utility function of cost versus gain of each user differs from user to user. What we should focus on research is to assess the tradeoff between the cost and the gain in user cooperative mobility.

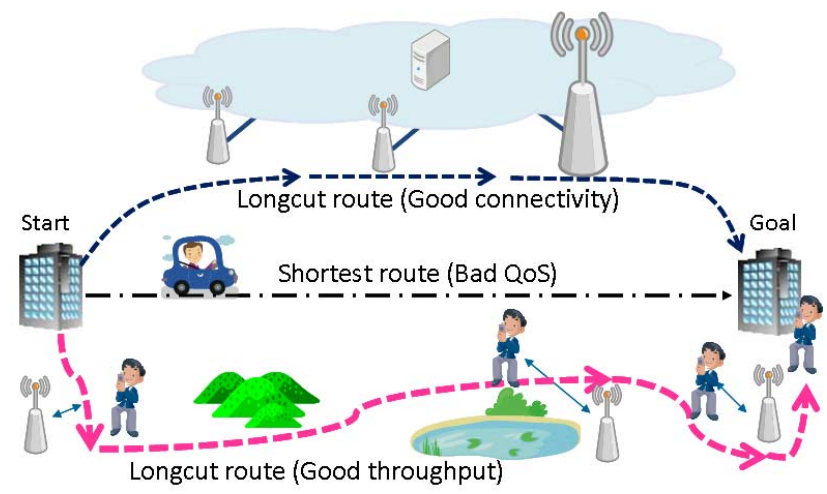

Figure 1. Longcut route

There are several research results reveal the tradeoff and justification of the usefulness of the user cooperative mobility. Wireless LANs can provide high speed access link but has a limited communication area (hot spot). Its throughput depends on the distance between terminals and access point. It is important for mobile users who move from A to B to know which route has better throughput as well as WLAN connectivity. The optimal longcut route is shown in [1] as in Fig. 1, which suggests candidate routes that have different pairs of throughput and real distance from A to B (see Fig. 2). Mobile users can select the route depending on their utility functions. Such user cooperation might prevent network operators from extra cost of adding access points so that this mobility is called "cooperative" [2]. In the hot spot, users can obtain more throughput as less distance to the AP. When mobile users can move toward the AP, they could be happy with more throughputs (Fig. 3). Performance anomaly in wireless LANs leverage the problem more complicated [4]. 


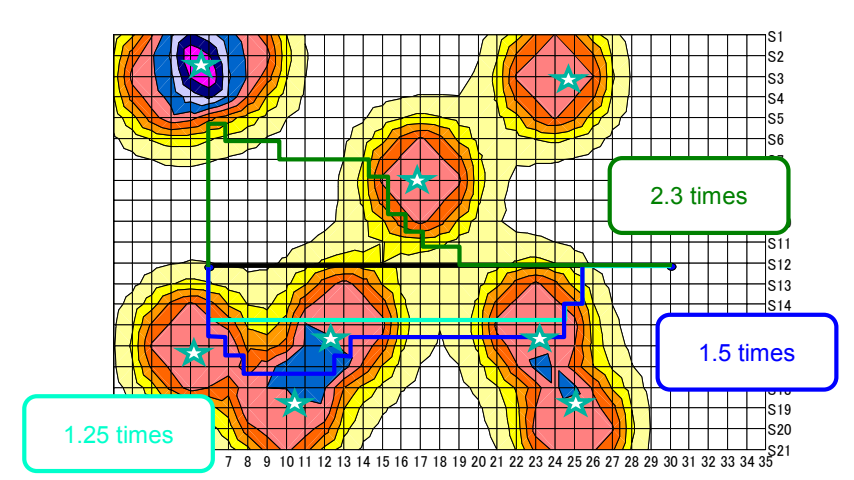

Figure 2. Optimal longcut route within cost limitation

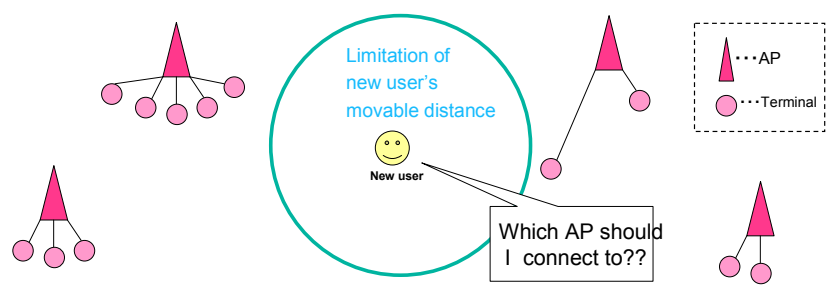

Figure 3. AP selection with user cooperative mobility

\section{Speaker Biography}

Tutomu Murase was born in Kyoto, Japan in 1961.

He received his M.E. degree from Graduate School of Engineering Science, Osaka University, Japan, in 1986. He also received his $\mathrm{PhD}$ degree from Graduate School of Information Science and Technology, Osaka University in 2004. He joined NEC Corporation Japan in 1986 and has been engaged in research on traffic management for high-quality and high-speed internet. His current interests include transport and session layer traffic control, wireless network resource management and network security. He also interested in user cooperative mobility research. $\mathrm{He}$ is a visiting professor of Tokyo Institute of Technology, Japan and is also a Principal Researcher in NEC Corporation Japan. He is the secretary of IEEE Communications Society Japan Chapter. $\mathrm{He}$ is a member of IEEE and a fellow of IEICE.

He received Best Tutorial Paper Award on his invited paper in IEICE transaction on communication in 2006. He has been serve as TPC for IEEE ICC, Globecom, CCNC, APCC, ICCCN, MMNS, in 2006-2013, 2006-2013,2009-2012, 2010-2013, 2006, 2005 , respectively. He has been serve as TPC for IEEE workshop such as CQR workshop, 2008-2013, SocNet workshop (in IEEE Globecom Workshop), 2010-2011, SIMNA(IEEE ICCCN Workshop in Social Interactive Media Networking and Application), 2011. He was a TPC in ACM ICUIMC (ITcom), 2012-2013. He has more than 90 registered patents including some international patents.

\section{REFERENCES}

[1] Motoyoshi, G., Sudo, Y., Murase, T. and Masuzawa, T. Advantages of Optimal Longcut Route for Wireless Mobile Users. In Proceedings of IEEE International Conference on Communications ICC2011. June 2011.

[2] Murase, T., Kakehi, T., Motoyoshi, G., Yamori, K. and Shinkuma, R. Optimal route planning in wireless networks for mobile users with incentive mechanism. In Proceedings of IEEE CCNC 2011. PP. 1114-1115, Jan. 2011.

[3] Kobayashi, K. and Matsunaga, Y. Radio Quality Prediction Based on User Mobility and Radio Propagation Analysis. In Proceedings of IEEE PIMRC 2009, Sep. 2009.

[4] Miyata, S., Murase, T. and Yamaoka, K. Characteristic analysis of an access-point selection for user throughput and system optimization based on user cooperative moving. In Proceedings of IEEE CCNC2012. Jan. 2012. 